\title{
Physiotherapy in rehabilitation and prohabilitation across the lifespan
}

\author{
N Naidoo, ${ }^{1}$ MMedSci, MEd, DMD, PhD; R Barnes, ${ }^{2} \mathrm{PhD} ; \mathbf{N}$ Mlenzana, ${ }^{3} \mathrm{PhD} ; \mathbf{K}$ Mostert,${ }^{4} \mathrm{PhD} ; \mathbf{S}$ L Amosun, ${ }^{1} \mathrm{PhD}$ \\ ${ }^{1}$ Division of Physiotherapy, Department of Health and Rehabilitation Sciences, Faculty of Health Sciences, University of Cape Town, South Africa \\ ${ }^{2}$ Department of Physiotherapy, School of Allied Health Professions, Faculty of Health Sciences, University of the Free State, Bloemfontein, \\ South Africa \\ ${ }^{3}$ Department of Physiotherapy, Faculty of Community and Health Sciences, University of the Western Cape, Cape Town, South Africa \\ ${ }^{4}$ Department of Physiotherapy, Faculty of Health Sciences, University of Pretoria, South Africa
}

Corresponding author: N Naidoo (niri.naidoo@uct.ac.za)

\begin{abstract}
There is considerable evidence to support the rise in non-communicable diseases (NCDs). Reduction of the NCD burden is of vital importance globally, including South Africa (SA). Recent evidence demonstrates that NCDs commence in early childhood and continue throughout the lifespan. Strengthening of interprofessional and multidisciplinary team efforts supports a decrease in the impact of NCDs on individuals, families and communities and enhances health-related quality of life to improve productivity of the SA economy. Mounting overweight/obesity rates and poor nutrition cause NCDs, which are preventable. It is important that healthcare practitioners identify causes of ill-health and promote health among patients, rather than await symptoms and then determine treatment. It is also critically important to inspire positive lifestyle changes in one's patients.
\end{abstract}

S Afr Med J 2019;109(3):142-144. DOI:10.7196/SAMJ.2019.v109i3.13902

Currently, the global population is 7.3 billion, which is projected to increase to 8.5 billion by 2030 and 9.7 billion by $2050{ }^{[1]}$ In 2017 , the estimated population of South Africa (SA) was 56 million people of diverse origins, cultures, languages and religions, with an estimated life expectancy of 64 years. ${ }^{[2]}$ The estimated prevalence of disability was $\sim 7 \%$. The country experiences a quadruple burden of diseases, i.e. HIV and AIDS, with a synergistic relationship with tuberculosis; maternal and child morbidity and mortality; non-communicable diseases (NCDs), mostly driven by risk factors related to lifestyle; and violence, injuries and trauma. ${ }^{[3]}$ As part of the post-apartheid transformation processes to correct past inequities, the government developed the National Development Plan (NDP) 2030. ${ }^{[4]}$ One of the goals of the NDP is to improve the health and wellbeing of the inhabitants across all population groups, thereby raising the average life expectancy to at least 70 years by 2030. Lifespan refers to the duration of life from conception until death.

The number of deaths from NCDs has increased worldwide and NCDs are a leading cause of death in many countries. While the World Health Organization (WHO) projected that by 2030 NCDs will be the largest cause of death in Africa, NCDs are currently among the top causes of death in SA. ${ }^{[5]}$ It is imperative that healthcare practitioners, as vital stakeholders, identify NCDs and promote health among our patients, rather than await the consequences before determining treatment. Health professionals also have a role to influence lifestyle changes in patients; this is a difficult task that is often instituted late.

Physical inactivity is a significant contributory but modifiable risk factor for NCDs. Physical activity (PA) is defined as any bodily movement produced by skeletal muscles that requires energy expenditure, and is not restricted to participation in sports and structured physical exercises. It is conceptualised as the relationship between human beings and their environments when moving the body by walking, running and other ways where people physically exert themselves regularly, whether at work, home, in transport to and from places or during leisure time. ${ }^{[6]} \mathrm{PA}$ is recommended as a cost-effective intervention to address the increasing burden of NCDs through health promotion and disease prevention at the community level.

Physiotherapy is the health profession most commonly associated with PA and physical function to promote and maintain health, as physiotherapists contribute to maximising quality of life within the spheres of promotion, prevention, treatment, habilitation and rehabilitation across the lifespan. ${ }^{[7]}$ Rehabilitation has been defined as 'a set of measures that assist individuals, who experience or are likely to experience disability, to achieve and maintain optimum functioning in interaction with their environments. ${ }^{[8]}$ Physiotherapy is instrumental in enabling people with limitations with regard to functioning to remain in or return to their home or community, live independently and participate in everyday life activities. For the purpose of this article, 'prohabilitation' is defined as the process of using exercise to advance and maintain optimal physical and mental fitness throughout one's lifespan.

In view of the impact of NCDs in $\mathrm{SA},{ }^{[3]}$ and 'rehabilitation across the lifespan' being one of the six priority areas for rehabilitation research, ${ }^{[9]}$ the purpose of this narrative overview is to provide evidence relating to the impact of physiotherapy on the management of NCDs to enhance health-related quality of life across the lifespan.

\section{Risk factors for non-communicable diseases}

Risk factors for NCDs are set during early development owing to exposure to nutrients and environmental chemicals, which may have profound effects on organ function and disease risks in later life. ${ }^{[10]}$ Typical risk factors include smoking, alcohol consumption, excess body weight and dietary factors that are responsible for a large share of the global disease burden, directly or by conditions such as high 
blood pressure and elevated blood glucose and cholesterol levels. ${ }^{[11]}$ $\mathrm{PA}$ is acknowledged for its role in the prevention and management of NCDs.

\section{Physical activity interventions Childhood}

Several studies have explored the impact of PA and diet on obesity in children and adolescents. It is unclear whether children are overweight/obese owing to a lack of PA or whether they do not participate in adequate PA because they are obese. Other contributors to decreased PA levels in childhood may include poor motor control and motor skill that discourage participation in sport and other activities. Children may have deficiencies in fundamental motor skills, knowledge and PA behaviours, which are essential requirements to lead healthy active lifestyles. Physical inactivity in childhood is associated with obesity and declining physical fitness levels. The relationship between motor proficiency and physical fitness requires consideration, as children with motor deficiencies are less likely to participate in PA. Additionally, compared with their high motor-competent counterparts, those with low motor competence are significantly less physically active. ${ }^{[12]}$ Moreover, typically developing (TD) children with movement difficulties, when compared with healthy controls who were age- and gender-matched, were found to be less vigorously active. ${ }^{[13]}$

The American Academy of Pediatrics recommends that children should be physically active for at least 60 minutes per day, as physical inactivity contributes to a high prevalence of overweight. ${ }^{[9]}$ The WHO proposes a similar recommendation to improve cardiorespiratory and muscular fitness, bone health and cardiovascular and metabolic health biomarkers. ${ }^{[1]}$ The WHO further recommends that children and youth aged 5 - 17 years should accrue at least 60 minutes of moderate to vigorous-intensity PA daily - mainly aerobic activity. Adults should accumulate at least 150 minutes of moderate aerobic activity such as cycling or brisk walking every week and strength exercises on $\geq 2$ days a week, working all the major muscles (legs, hips, back, abdomen, chest, shoulders and arms). The incorporation of vigorous-intensity activities, including those that strengthen muscles and improve bone heath, are recommended at least 3 times per week.

\section{People with disabilities}

The prevalence of increased body mass index (BMI) in people with disabilities, ranging from children through to adulthood, is on the rise. Evidence that people with cerebral palsy (CP) have a higher prevalence of cardiometabolic risk factors such as hypertension, hyperlipidaemia and obesity is inconsistent; however, there is strong evidence that they participate in low levels of PA compared with those without $\mathrm{CP}^{[14]}$ Adults with $\mathrm{CP}$ have a higher risk of NCDs, including stroke, chronic obstructive pulmonary disease and other cardiovascular conditions; also death due to NCDs, including cancer, chronic obstructive pulmonary disease, stroke and ischaemic heart disease compared with the general population. ${ }^{[14]}$ The burden of NCDs is therefore higher among adults with $\mathrm{CP}$ than in the general population.

A review of PA in children with disabilities reported that fewer hours were spent on moderate and high levels of PA compared with TD children ${ }^{[15]}$ Regular participation in PA by children, including those with intellectual disability, enhances body composition, bone health and psychological health and promotes social engagement. Key facilitators for PA participation are enjoyment and unstructured activities, social interactions, parental support and increasing levels of independence. Such facilitators may have beneficial effects for PA participation in later life.

\section{Musculoskeletal conditions}

Musculoskeletal conditions impact negatively on physical health and there is evidence that individuals with these conditions and joint pain are more likely to experience activity limitations - in the short and long term. There is also a strong association between musculoskeletal pain and decreased PA and being overweight. The resulting combination may increase the pain associated with musculoskeletal conditions and add to the stress of daily life, making even simple tasks difficult and affecting the cognitive ability of the individual. With the increasing longevity of the population, it is likely that the prevalence of musculoskeletal pain will continue to rise. The prevalence of musculoskeletal conditions is higher in women than in men and increases with age.

Many studies have been conducted with the aim of increasing $\mathrm{PA}$, especially in adults, for the management of joint pain, and have demonstrated that PA interventions ameliorate such pain. Therapeutic programmes should be tailored according to the needs of the community and consider the population for which the programme is developed. Self-efficacy, goal-setting and community involvement should be encouraged in practice to ensure the sustainability of any intervention programme that encourages increased PA or exercise. There appears to be definite merit in education programmes to enhance the health-related quality of life of individuals with joint pain as well as the Enabling Self-management and Coping with Arthritic Pain using Exercise (ESCAPE) knee pain programme that improved the physical function of the participants with chronic knee pain in the short term. ${ }^{[16]}$

\section{Older persons}

In 2017, the number of people aged $\geq 60$ years in SA was estimated to be 4.6 million of a population of 56 million. ${ }^{[2]}$ The Global Age Watch Index (GAWI) assessed the impact of ageing policies in 195 countries by taking into account contextual, structural and cultural factors. ${ }^{[6]}$ Of 96 countries that were ranked, the top 5 in 2015 were Switzerland, Norway, Sweden, Germany and Canada. Africa was over-represented at the bottom of the ranked countries, with Mauritius and SA being the first two African countries, but respectively listed in 42nd and 78th positions. Despite the socioeconomic differences between the leading countries on the GAWI list and SA, PA remains a costeffective intervention in all settings in older persons.

Older persons with chronic health conditions have relatively high attendance rates at general practitioners (GPs)' rooms. GPs, therefore, are in a strong position to give exercise-related advice, although there is little or no evidence that this is common practice in SA. While living longer should be celebrated, maintenance of functional independence is also of high importance to maintain quality of life. WHO guidelines and global recommendations on PA for health also include proposals for older persons. ${ }^{[17]}$ The health benefits of regular participation in either supervised or unsupervised (e.g. gardening, home activities, walking, stair climbing) PA programmes have been reported. Indoor walking programmes are popular among older persons in Canada because of the severe winter weather. An 8-week pilot programme that assessed the feasibility of community mall walking, improved the fitness level of participants, whose average age was 66.4 years. Significant changes were reported in the BMI, waist-to-hip ratio, resting heart rate, walk-test distance, walk-test heart rate, post-walk test rate of perceived exertion and flexibility. Neighbourhood dog walking close to the homes of older persons also resulted in increased PA levels. ${ }^{\left[{ }^{6]}\right.}$ Exercise at a moderate level reduced all-cause mortality and risk of developing conditions such as cardiovascular disease and type 2 diabetes in the elderly. Also, 
participation in exercise of sufficient intensity and frequency reduced the risk of functional limitation and disability.

Promotion of and participation in PA in the older population determine their health-related quality of life and how they experience being physically active. Factors such as socioeconomic status, culture, age and sex-specific roles and responsibilities affect participation in PA in the older population. In a multi-country population-based study, the association of low socioeconomic status and risk factors for NCDs (diabetes, high alcohol intake, high blood pressure, obesity, physical inactivity, smoking) with loss of physical functioning at an older age was assessed. The investigators concluded that the independent association between socioeconomic status and physical functioning in old age is comparable in strength and consistency with that for established NCD risk factors, ${ }^{[18]}$ suggesting that intervening in all these risk factors might substantially increase life years spent in good physical functioning.

\section{Interdisciplinary and community- based care}

Attempts to raise participation in PA across one's lifespan require introducing interdisciplinary and community-based care. As the majority of patients are known to encounter problems in accessing rehabilitation services owing to challenges related to access to transport, shortage of resources and lack of information on available programmes and services, it is important to consider implementation of interventions at community level. Community-based services include home- and community-based care, intermediate care and primary care services at health facilities and non-medical sites. Healthcare 2020 shifted the focus from mere treatment to the prevention of disease. The plan was based on improving accessibility to healthcare by expanding community- and home-based services, services that focus on prevention, promotion of wellness, curing of disease/illness and a patient-centred approach to rehabilitation. The last was thought to encourage interaction between rehabilitation professionals and the patient.

Interprofessional collaboration improves healthcare and the delivery of service by teaching individuals from different professions to work better together and learn from each other. Interprofessional practice has been identified as a vehicle to address the health and social needs of our society. Primary healthcare service is the most critical component, as it serves as the admission into the care field and caters for the vast majority of patient contacts. Support, information sharing, communication, environmental factors, time and referral systems were considered to be important for improving rehabilitation and prohabilitation services. Community-based physiotherapists can be pivotal in effectively promoting PA in different non-clinical settings, working with clients from across the lifespan.

Parallel to the reorientation of primary healthcare in SA, physiotherapists work in community-based settings and are ideally positioned to provide health education, including PA prescription, and support essential work as part of an interprofessional team. As part of routine medical care, patients are referred for PA prescription and/or counselling; physiotherapists thus assist sedentary patients to exercise, and those who are already active to exercise more. The main reasons for referral for exercise prescription in the primary care setting are sedentary lifestyle, musculoskeletal impairments, overweight, diabetes, hypertension, abnormal cholesterol levels and mental health.

Community-based physiotherapists can be pivotal in effectively promoting PA in different non-clinical settings, working with clients from across the lifespan. In addition to PA programmes for the general public and the prevention of chronic diseases, persons with chronic conditions (such as osteoarthritis), as well as those with other long-term disabilities (such as stroke) and mental illness, can benefit from community-based opportunities for PA (such as hydrotherapy). Maintenance of these interventions is often achieved, although not always reported. There is an urgent need for healthcare professionals to empower patients with knowledge of the health advantages of PA. In that way they may be agents of change for themselves, their families, communities and ultimately for the economy.

\section{Conclusion}

We have highlighted the role of physiotherapy and PA in multisectoral approaches to prevention and control of the burden of NCDs in SA. Evidence supports PA as having the potential, across the lifespan, to reduce obesity and improve several health outcomes, including arthritis, diabetes, cancer and cardiovascular disease. However, in SA, as in many other countries, safety remains an obstacle to participation in PA at community level. While participation in PA should be encouraged, it is important to also ensure that the policies and programmes that are developed foster safe places in communities for regular PA.

\section{Declaration. None.}

Acknowledgements. None.

Author contributions. The article was conceived by the primary author and co-ordinated by the primary author and the other authors. All authors contributed to the respective sections.

Funding. None.

Conflicts of interest. None.

1. United Nations. World Population Prospects: Key findings and advance tables. 2015. https://esa. un.org/unpd/wpp/publications/files/key_findings_wpp_2015.pdf (accessed 22 January 2019).

Statistics South Africa. Mid-year Population Estimates. Pretoria: Stats SA, 2017. 2. Statistics South Africa. Mid-year Population Estimates. Pretoria: Stats SA, 2017.
3. Mayosi MB, Benatar SR. Health and health care in South Africa - 20 years after Mandela. N Engl J Med
2014:371:1344-1353. https://doi.org/10.1056/NEJMsr1405012

2014;371:1344-1353. https://doi.org/10.1056/NEJMsr1405012
4. South African Government. National Development Plan 2030. Pretoria: Government Printer, 2011:330-351

5. Nojilana B, Bradshaw D, Pillay-van Wyk V, et al. Emerging trends in non-communicable disease mortality in South Africa, 1997 - 2010. S Afr Med J 2016;106(5):477-484. https://doi.org/10.7196/ SAMJ.2016.v106i4.10674

6. Toohey AM, McCormack GR, Doyle-Baker PK, Adams CL, Rock MJ. Dog-walking and sense of community in neighbourhoods: Implications for promoting regular physical activity in adults 50 year and older. Health Place 2013;22:75-81.

7. Wickford J, Duttine A. Answering global health needs in low-income countries: Considering the role of physical therapists. World Med Health Policy 2013;5(2):141-160.

8. World Health Organization. World Report on Disability. Geneva: WHO, 2011.

9. Jette AM. Moving rehabilitation research forward at NHI. Phys Ther 2017;97(4):389. https://doi. org/10.1177/1747493016643851

10. Barouki R, Gluckman PD, Grandjean P, Hanson M, Heindel JJ. Developmental origins of noncommunicable disease: Implications for research and public health. Environ Health 2012;11:42. communicable disease: Implications for

11. Ezzati M, Riboli E. Behavioral and dietary risk factors for noncommunicable diseases. N Engl J Med Ezzati M, Riboli E. Behavioral and dietary risk factors for
2013;369:954-964. https://doi.org/10.1056/NEJMra1203528

12. Wall A. The developmental skill-learning gap hypothesis: Implications for children with movement difficulties. Adapt Phys Activity Quart 2004;21(3):197-218.

13. Ryan JM, Allen E, Gormley J, Hurvitz EA, Petersen MD. The risk, burden, and management of non-communicable diseases in cerebral palsy: A scoping review. Develop Med Child Neurol 2018;60(8):753-764. https://doi.org/10.1111/dmcn.13737

4. Hinckson E, Curtis A. Measuring physical activity in children and youth living with intellectual disabilities: A systematic review. Res Develop Disabil 2013;34(1):72-86.

15. Hurley MV, Walsh NE, Mitchell H, Nicholas J, Patel A. Long-term outcomes and costs of an integrated rehabilitation program for chronic knee pain: A pragmatic, cluster randomized, controlled trial. Arthr Care Res 2010;64(2):238-247. https://doi.org/10.1002/acr.20642

16. Help Age International. Global Age Watch Index. 2015. http://www.helpage.org/global-agewatch/ pelp Age International. Global Age Watch Index. 2015. http://www.
population-ageing-data/global-rankings-table/ (accessed 10 June 2018).

17. World Health Organization. The Global Economic and Healthcare Burden of Musculoskeletal Disease.
population-ageing-data/global-rankings-table/ (accessed 10 June 2018). Geneva: WHO, 2010:1-6.

18. Evans AB, Nistrup A, Allen-Collinson J. Socio-cultural approaches to ageing: Changing our understanding of the life-course? In: Nyman S, ed. The Palgrave Handbook of Ageing and Physical Activity Promotion. London: Palgrave Macmillan, 2018:551-569. 